\title{
Early Mobilization and Rehabilitation in the ICU: Moving Back to the Future
}

\author{
Mohamed D Hashem MD, Archana Nelliot, and Dale M Needham MD PhD
}

\author{
Introduction \\ Historical Background \\ Effects of Bed Rest \\ ICU-Acquired Weakness \\ Safety and Feasibility of Early Mobilization and Rehabilitation \\ Evidence for Effect on Patient Outcomes \\ Steps to Close the Gap Between Research and Practice \\ Practical Experience From the Johns Hopkins Hospital \\ Future Directions for the Field \\ Conclusions
}

\begin{abstract}
Despite the historical precedent of mobilizing critically ill patients, bed rest is common practice in ICUs worldwide, especially for mechanically ventilated patients. ICU-acquired weakness is an increasingly recognized problem, with sequelae that may last for months and years following ICU discharge. The combination of critical illness and bed rest results in substantial muscle wasting during an ICU stay. When initiated shortly after the start of mechanical ventilation, mobilization and rehabilitation can play an important role in decreasing the duration of mechanical ventilation and hospital stay and improving patients' return to functional independence. This review summarizes recent evidence supporting the safety, feasibility, and benefits of early mobilization and rehabilitation of mechanically ventilated patients and presents a brief summary of future directions for this field. Key words: ICU; rehabilitation; early mobilization; bed rest; physical therapy; occupational therapy; mechanical ventilation. [Respir Care 2016;61(7):971-979. (C) 2016 Daedalus Enterprises]
\end{abstract}

\section{Introduction}

Early mobilization and rehabilitation of mechanically ventilated patients in the ICU is a topic of growing interest. This review will summarize recent evidence on safety,

\footnotetext{
The authors are affiliated with the Outcomes after Critical Illness and Surgery Group and the Division of Pulmonary and Critical Care Medicine, School of Medicine, Johns Hopkins University, Baltimore, Maryland. Dr Needham is also affiliated with the Department of Physical Medicine and Rehabilitation, School of Medicine, Johns Hopkins University, Baltimore, Maryland.

Dr Needham presented a version of this paper as the 3rd Annual Thomas L Petty Memorial Lecture at the 61st AARC Congress, held November 7-10, 2015, in Tampa, Florida.
}

feasibility and potential benefits of early mobilization and rehabilitation, in addition to highlighting some future directions for this field.

\section{Historical Background}

Reports of mobilizing hospitalized patients have been published since the late 19th century. For instance, in a

\footnotetext{
Correspondence: Dale M Needham MD PhD, Pulmonary and Critical Care Medicine, Johns Hopkins University, 1830 E Monument St, 5th Floor, Baltimore, MD 21205. E-mail: dale.needham@jhmi.edu
}

DOI: $10.4187 /$ respcare. 04741 
publication from $1899,{ }^{1}$ there was discussion of a "radical change in the after-treatment of celiotomy cases." This publication recognized that the postoperative period of bed rest could be cut to hours, instead of days or weeks, and result in reduced muscle weakness. Similar findings were published in subsequent years for patients recovering from other types of surgery and for women in the postpartum period. $2-4$

During World War II, these same concepts were employed to help injured soldiers return to the battlefield more quickly. ${ }^{5,6}$ An early controlled clinical trial to evaluate the effectiveness of early mobility after major surgery was published in $1944 .^{7}$ This trial described early mobility in 100 consecutive subjects compared with another 100 subjects who had usual care. After similar surgeries, the early mobility subjects sat in a chair and walked on the first day after surgery, whereas the other group was confined to bed rest for 10-15 days as part of usual care. The total number of complications, including local surgical, pulmonary, cardiac, vascular, genitourinary and gastrointestinal complications, was 17 versus 46 in the intervention versus control groups, and no major complications (eg, pulmonary emboli or coronary thrombosis) occurred in the early mobility group. ${ }^{7}$

In 1944, a conference on bed rest was held, ${ }^{6}$ and major journals were publishing on related topics, such as the "evil sequelae of complete bed rest" and the "abuse of rest in bed" 8,9 around this time. After the subsequent creation of ICUs, there were reports of the benefits of early mobilization in mechanically ventilated patients. ${ }^{10,11}$ Thomas Petty, a leader in pulmonary and critical care medicine, highlighted historical practices from the early days of critical care, in contrast to later practice, by saying: "When we first started our unit in 1964, patients who required mechanical ventilation were awake and alert and often sitting in a chair... But what I see these days are paralyzed, sedated patients, lying without motion, appearing to be dead, except for the monitors that tell me otherwise." 12 Thus, there is a strong historical basis for early mobilization and rehabilitation of hospitalized patients, including mechanically ventilated patients in the ICU.

\section{Effects of Bed Rest}

Bed rest can lead to rapid deconditioning and muscle atrophy. ${ }^{13}$ Studies of young healthy adults have shown that after 2 weeks of immobilization, there is a 5-9\% loss of quadriceps muscle mass and $20-27 \%$ decrease in quadriceps muscle strength. ${ }^{14,15}$ These effects are often accelerated and more pronounced in older adults, with a 3-6-fold greater rate of muscle loss. ${ }^{16,17}$

In mechanically ventilated patients, skeletal muscle cross-sectional area can decrease by $12.5 \%$ over the first week in the ICU. ${ }^{18}$ In ventilated patients with multiple- organ failure, muscle loss is much greater compared with those with only single-organ failure $(8.7 \%$ vs $1.8 \%$ after $3 \mathrm{~d}$, and $15.7 \%$ vs $3.0 \%$ after $7 \mathrm{~d}$ of ICU stay). ${ }^{18}$ Muscle biopsies from mechanically ventilated patients show signs of inflammation, necrosis, and replacement of muscle fibers with adipose and connective tissue. ${ }^{18,19}$

Bed rest in the ICU may be an important risk factor for long-term muscle weakness. A prospective study longitudinally following 222 ARDS survivors at 3, 6, 12, and 24 months reported a 3-11\% relative decrease in muscle strength for every additional day of bed rest in the ICU, after adjusting for other potential risk factors that may contribute to long-term weakness. ${ }^{20}$ Other physical outcome measures, including 6-min walk distance and quality of life scores, remained consistently lower than population norms through the 2-y follow-up period, with worse results in those with versus without muscle weakness. ${ }^{20}$

Therefore, bed rest can be an important risk factor for weakness in ICU patients. Early recognition of this issue is a key step in improving patient outcomes.

\section{ICU-Acquired Weakness}

ICU-acquired weakness is defined as the presence of clinically detectable weakness in ICU patients with no possible etiology other than critical illness. ${ }^{21}$ Clinically detectable weakness is generally evaluated via a standardized physical examination of strength, known as manual muscle testing, using the ordinal 6-point Medical Research Council scale (ranging from 0 [no palpable or visible muscle contraction] to 5 [normal strength]). Traditionally, manual muscle testing is performed in 3 muscle groups in each extremity bilaterally, with weakness diagnosed as a total score of $<48$ of $60 .{ }^{22,23}$ This approach to evaluating strength has its limitations, including the need for patients to be awake, cooperative, and capable of actively moving the extremities. ${ }^{21,24}$ Although there is large variability in its reported prevalence, more than one third of patients requiring mechanical ventilation for at least $5 \mathrm{~d}$ may have ICU-acquired weakness. ${ }^{25}$

Such weakness of the extremities as occurs with ICUacquired weakness is also associated with respiratory muscle weakness and prolonged weaning from mechanical ventilation. ${ }^{26-28}$ Consequently, recognition of ICU-acquired weakness may be important due to patients' increased risk of ventilator-associated pneumonia and recurrent respiratory failure. ${ }^{29}$

A prospective study compared 122 subjects with ICUacquired weakness in a mixed medical/surgical ICU with propensity-matched controls, finding that ICU-acquired weakness was independently associated with a longer duration of mechanical ventilation ( $11 \mathrm{~d}$ vs $8 \mathrm{~d}, P=.009)$ and hospital stay ( $36 \mathrm{~d}$ vs $23 \mathrm{~d}, P=.007$ ), greater total costs per patient $(\$ 23,277$ vs $\$ 17,834, P=.040)$, and 
increased 1 -y mortality (30.6\% vs $17.2 \%, P=.02)$. Furthermore, subjects with persistent and severe weakness (ie, Medical Research Council score $<36$ of 60 at the end of ICU stay) had a higher risk of death over 1-y follow-up (hazard ratio 4.3, $P<.001$ ). ${ }^{28}$

ICU survivors with ICU-acquired weakness also experience significant long-term impairment in respiratory muscle strength, physical functioning, and quality of life, lasting for months and years after hospital discharge. ${ }^{20,30-32} \mathrm{As}$ outlined below, early mobilization and rehabilitation of critically ill patients may play an important role in preventing these sequelae.

\section{Safety and Feasibility of Early Mobilization and Rehabilitation}

Given the growing literature on ICU-acquired weakness and the harms of bed rest, early mobilization and rehabilitation of critically ill patients is regaining attention. Despite the potential concerns about mobilizing mechanically ventilated patients, many studies have repeatedly demonstrated its safety and feasibility, with very low rates of potential safety events. In a German national point prevalence study of 775 mechanically ventilated subjects, the frequency of potential safety events was no higher with out-of-bed versus in-bed activity. ${ }^{33}$ With mobilization of mechanically ventilated patients, the most frequent potential events are physiological changes that are usually transient and resolve after rest, without any intervention. ${ }^{34-36}$

Recently, a panel of experts agreed that endotracheal intubation should not be a contraindication to active in-bed or out-of-bed mobilization or rehabilitation in the ICU setting. ${ }^{37}$ This report provided relevant safety guidelines, categorized by each body system (eg, respiratory, cardiovascular, and neurological systems), for mobilizing critically ill patients. Figure 1 demonstrates an example of the panel's recommendations regarding respiratory safety criteria. ${ }^{37}$

Feasibility of early mobility for mechanically ventilated patients is well recognized. A study of 103 mechanically ventilated subjects admitted over a 6-month period assessed safety and feasibility of early progressive mobility, including sitting on the edge of the bed, sitting in a chair, and ambulating. Of 1,449 mobility sessions, $41 \%$ involved intubated subjects, with 249 events in which intubated subjects ambulated, and $<1 \%$ occurrence of potential safety events. ${ }^{36}$ Figure 2 is a photograph of a patient ambulating while receiving mechanical ventilation via an endotracheal tube, with assistance from respiratory and physical therapists.

A bundle has been proposed that combines early mobility with awakening/breathing coordination (ie, spontaneous awakening trials with cessation of any infusion of sedative agents, combined with spontaneous breathing trials) and delirium monitoring/management (the ABCDE

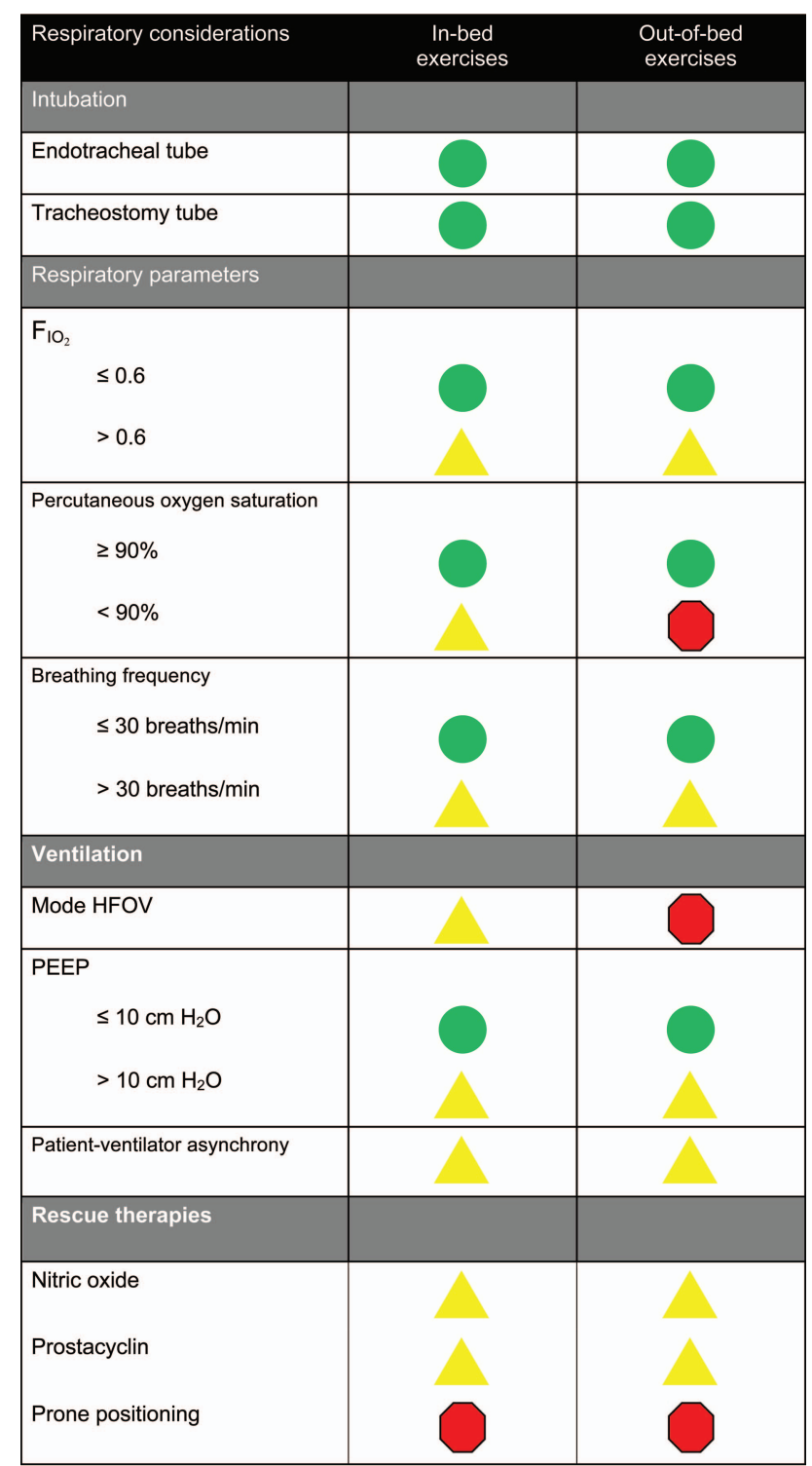

Fig. 1. Respiratory safety considerations. Green: Low risk of an adverse event. Proceed as usual according to each ICU's protocols and procedures. Yellow: Potential risk and consequences of an adverse event are higher than green but may be outweighed by the potential benefits of mobilization. The precautions or contraindications should be clarified before any mobilization episode. If mobilized, consideration should be given to doing so gradually and cautiously. Red: Significant potential risk or consequences of an adverse event. Active mobilization should not occur unless specifically authorized by the treating intensive care provider in consultation with senior physical therapy and nursing staff. From Reference 37.

bundle). ${ }^{38}$ Implementing the bundle in a pre-post study of 296 subjects (187 mechanically ventilated) showed that subjects in the post- versus pre-implementation period had more ventilator-free days (median of $24 \mathrm{~d}$ vs $21 \mathrm{~d}, P=.04$ ). After adjusting for age, severity of illness, comorbidity, and mechanical ventilation status, subjects in the post- 




Fig. 2. A patient ambulating while receiving mechanical ventilation via endotracheal tube, with the assistance of a respiratory therapist (front) and a physical therapist (behind). With patient and staff permission.

versus pre- group were more likely to mobilize out of bed at least once during an ICU stay (odds ratio $2.11, P=.003$ ) and less likely to experience delirium at any time in their ICU stay (odds ratio $0.55, P=.03$ ). ${ }^{39}$ Hence, a coordinated multidisciplinary approach can be effective in overcoming the barriers of deep sedation and delirium, allowing patients to benefit from early mobilization.

\section{Evidence for Effect on Patient Outcomes}

Early rehabilitation of mechanically ventilated patients may have both short- and long-term benefits. A nonrandomized, controlled trial assigned 280 mechanically ventilated subjects to either receive usual care or a mobility protocol that included 4 levels of activities, ranging from passive range of motion to active transfer to a chair. The protocol was conducted by a dedicated mobility team (critical care nurse, nursing assistant, and physical therapist) $7 \mathrm{~d} /$ week, starting within $48 \mathrm{~h}$ of mechanical ventilation. After adjusting for body mass index, Acute Physiology and Chronic Health Evaluation II (APACHE II) score, and vasopressors, subjects in the intervention group got out of bed much earlier (5.0 vs $11.3 \mathrm{~d}, P<.001)$ and had a shorter stay in the ICU $(5.5 \mathrm{~d}$ vs $6.9 \mathrm{~d}, P=.02)$ and hospital (11.2 d vs $14.5 \mathrm{~d}, P=.006) .{ }^{40}$ A subsequent follow-up study showed that lack of early mobility was independently associated with a higher odds of death or readmission within $1 \mathrm{y}$ of hospitalization (odds ratio $=1.77$, 95\% CI $=1.04-3.01, P=.036) .{ }^{41}$

In a randomized controlled trial conducted in 2 university hospital ICUs, 104 mechanically ventilated subjects were randomized to either receive usual care or early physical therapy and occupational therapy interventions. Sub- jects randomized to early physical therapy and occupational therapy interventions were more likely to return to independent physical functioning at hospital discharge (59\% vs $35 \%, P=.02$ ), have shorter duration of mechanical ventilation ( $3.4 \mathrm{~d}$ vs $6.1 \mathrm{~d}, P=.02)$, and have fewer days with delirium in the ICU ( $2 \mathrm{~d}$ vs $4 \mathrm{~d}, P=.03)$. A key reason for these benefits was the early start to rehabilitation interventions. Specifically, the intervention versus control group started physical therapy and occupational therapy interventions at $1.5 \mathrm{~d}$ versus $7.4 \mathrm{~d}(P<.001)$ after intubation and had a much greater daily median duration of physical therapy/occupational therapy interventions while mechanically ventilated $(19 \mathrm{~min} / \mathrm{d}$ vs $0 \mathrm{~min} / \mathrm{d}$, $P<.001) .{ }^{42}$

On the other hand, a single-center randomized controlled trial randomized 150 subjects in the ICU for $\geq 5 \mathrm{~d}$ to usual care (ie, physical therapy available $7 \mathrm{~d}$ /week) versus an intensive exercise regimen in the ICU, ward, and outpatient clinic. This trial demonstrated no significant difference in patient outcomes over 12 months of followup. ${ }^{25}$ Another multi-center randomized controlled trial randomized 120 mechanically ventilated subjects to up to $28 \mathrm{~d}$ of physical therapy in the ICU and on the ward at $3 \mathrm{~d} /$ week (control group, with actual average duration per session of $22 \mathrm{~min}$ ) versus $7 \mathrm{~d} /$ week (intervention group, with actual average duration per session of $39 \mathrm{~min}$ ). The physical therapy intervention started at a median (interquartile range) of $8(6-11) \mathrm{d}$ after intubation. There was no significant difference in physical function at 1-, 3-, and 6-month follow-up, with the primary outcome only measureable in one third of patients at 1-month follow-up. ${ }^{43}$ In contrast to the trials with positive results, described above, both of these negative trials started the rehabilitation intervention relatively late after initiation of mechanical ventilation and had control groups with physical therapy delivered at a much higher intensity compared with control groups in the positive trials and compared with usual practice. ${ }^{35,44}$ More research, with larger sample sizes, should evaluate the optimal timing and dose of rehabilitation in the ICU. However, based on current evidence, initiation of rehabilitation early after intubation appears to improve patient outcomes compared with the usual practice in most ICUs of little or no rehabilitation during mechanical ventilation.

\section{Steps to Close the Gap Between Research and Practice}

Despite the well-known detrimental effects of bed rest and research supporting early initiation of rehabilitation, mobilizing ICU patients in routine clinical practice remains uncommon, especially for mechanically ventilated patients. In a recent point prevalence study of therapistdelivered mobility in 770 subjects from 33 ICUs within 


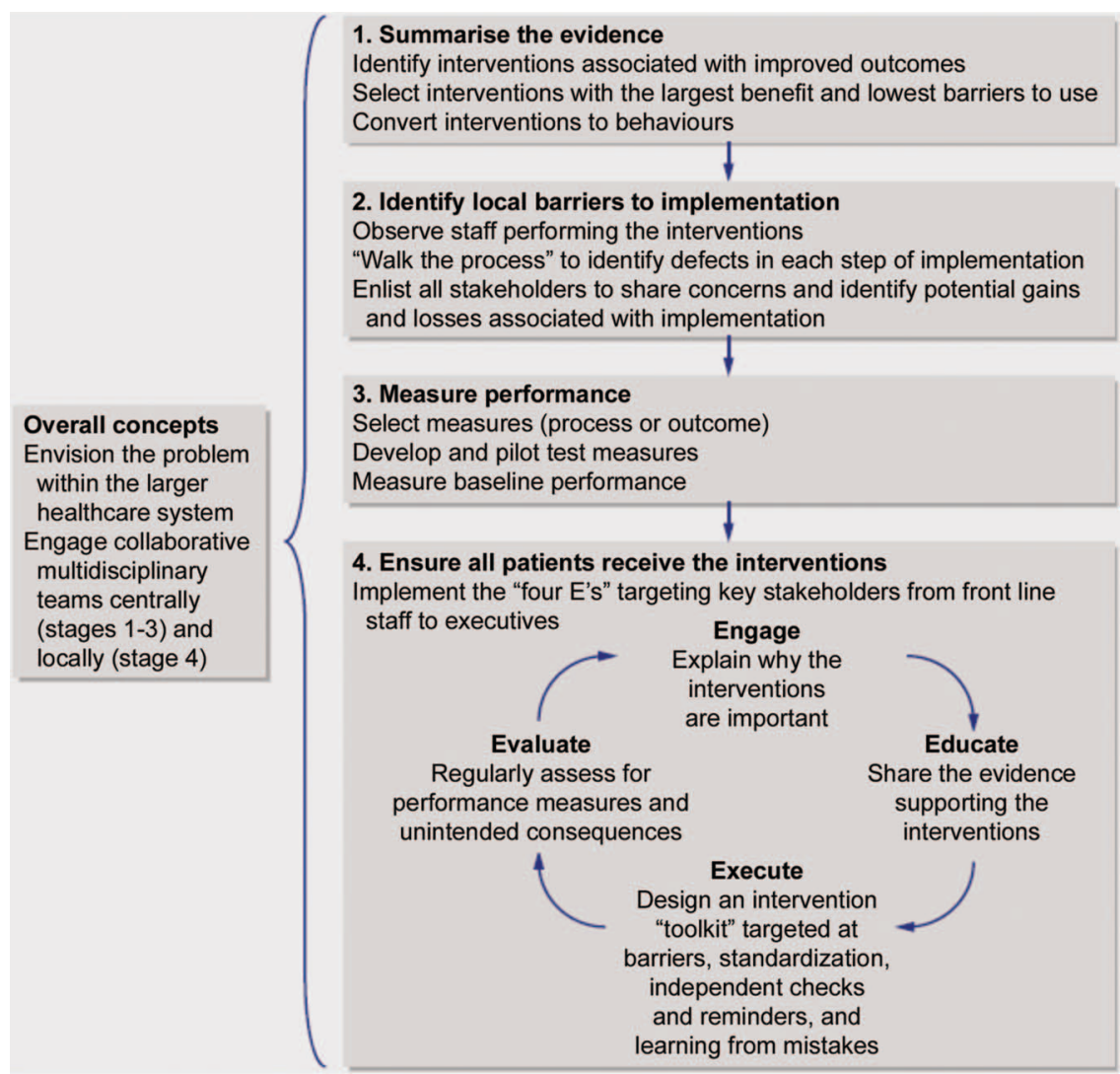

Fig. 3. Summary of a quality improvement model for translating research evidence into routine clinical practice to improve patient outcomes. From Reference 49, with permission.

the United States ARDS Network, sitting at the edge of bed or greater activity occurred in only $16 \%$ of mechanically ventilated subjects. ${ }^{44}$ Likewise, 2 national single-day point prevalence studies in Germany and Australia/New Zealand have been completed. The German study reported that, among 775 mechanically ventilated subjects, only $24 \%$ were at least sitting on the edge of the bed, ${ }^{33}$ whereas the Australia/New Zealand study reported that none of the 222 mechanically ventilated subjects sat out of bed or walked. ${ }^{35}$

A common barrier to mobilizing critically ill patients is inadequate staffing of physical and occupational therapists in ICUs. In the United States, physical therapists are infrequently available in ICUs, with a median (interquartile range) of 6.3 (4-10) physical therapists/100 ICU beds..$^{45}$ Only $34 \%$ of ICUs report having a dedicated physical therapy/occupational therapy team, and $30 \%$ have a written protocol for early mobilization. ${ }^{46}$

However, even with adequate staffing, mechanically ventilated patients may still be infrequently mobilized. For instance, in a prospective study of 192 mechanically ventilated subjects in Australia/New Zealand, where physical therapists also deliver respiratory therapy and there is a median of 1 physical therapist/9 ICU beds, $64 \%$ of sub- jects did not receive early mobilization, and $45 \%$ of all rehabilitation sessions were conducted with the subject in bed. ${ }^{47}$

Another Australian study of 106 ICU subjects reported that perceived barriers to mobilization (eg, femoral lines, timing of procedures, and sedation) were potentially avoidable in $47 \%$ of patient days where mobilization did not occur. 48

Successfully closing this gap between research and clinical practice requires the use of structured multistep quality improvement efforts. One such quality improvement approach is the Translating Research into Practice model. ${ }^{49}$ Within the Translating Research into Practice model, it is critical that a multidisciplinary team be engaged to evaluate the research-to-practice gap within the larger healthcare setting. The model consists of 4 steps (Fig. 3): (1) summarizing the evidence to understand the highest-yield intervention(s) that will address the health-care problem (eg, early mobility/rehabilitation to address physical impairments in critically ill patients); (2) identifying local barriers to the implementation of these interventions; (3) creating metrics or performance measures to evaluate progress with overcoming barriers and implementing the intervention; and (4) ensuring that all patients receive the in- 
tervention by using the " 4 Es" framework, which involves an iterative process of engaging stakeholders and then educating them before moving onward to executing the intervention and continuously evaluating it using the progress measures from Step 2.49,50 An example of executing this model took place in the form of a quality improvement project at the Johns Hopkins Hospital, as described in the next section.

\section{Practical Experience From the Johns Hopkins Hospital}

In the Johns Hopkins Hospital medical ICU, a multidisciplinary quality improvement project targeting early rehabilitation was planned over an 8-month period and then executed over 4 months. ${ }^{51}$ The quality improvement project, conducted using the Translating Research into Practice model, focused on all medical ICU patients, with detailed data collection and evaluation completed for patients requiring $\geq 4 \mathrm{~d}$ of mechanical ventilation, without any preexisting cognitive or neuromuscular problems. Among the steps included in executing the quality improvement project were modifying the default activity level in the medical ICU admission order set from "bed rest" to "activity as tolerated," changing sedation practice from continuous infusions to "as needed" boluses, providing guidelines for both physical therapy and occupational therapy consultations, and implementing safety screening guidelines for rehabilitation in ICU patients.

Compared with the 3-month period immediately preceding the quality improvement project, there was a significant decrease in the use of sedative medications, with a significant increase in the proportion of days in which patients were alert $(66 \%$ vs $29 \%, P<.001)$ and not delirious $(53 \%$ vs $21 \%, P=.003)$. In addition, there was a significant decrease in the proportion of ICU days in which eligible patients failed to receive rehabilitation therapy (7\% vs $41 \%, P=.004)$. Among 294 physical therapy and occupational therapy treatments given, there were only 4 $(1.4 \%)$ potential safety events that were minor in nature. Compared with the same 4-month period in the preceding year, the quality improvement period had a $30 \%$ decrease in the average medical ICU stay $(P=.02)$, with a $20 \%$ increase in the number of medical ICU admissions. ${ }^{51}$

Following the success of this quality improvement project, Johns Hopkins Hospital funded an early rehabilitation program that increased the full-time rehabilitation staff dedicated to the medical ICU. In addition, a new sedation protocol was created, and standardized delirium assessments by nurses became routine practice. ${ }^{50,52} \mathrm{~A}$ prospective cohort study evaluating sustainability of the quality improvement project compared data on consecutive ARDS subjects admitted in the 3 y preceding the quality improvement project with ARDS subjects admitted over a 3-y period starting $2 \mathrm{y}$ after completion of the quality improvement project. This comparison, extending out to $5 \mathrm{y}$ after completion of the quality improvement project, demonstrated that subjects in the post-quality improvement period had a shorter time to initiation of physical therapy (adjusted hazard ratio $=8.4,95 \%$ CI 5.0-14.1, $P<.001$ ) that was significant for each of the $5 \mathrm{y}$ during the postquality improvement period. In addition, in the post-quality improvement versus pre-quality improvement period, there was a significant increase in the proportion of subjects ever receiving physical therapy (68\% vs $16 \%, P<.001$ ) and achieving a higher daily activity level during physical therapy treatments (eg, sitting at the edge of the bed, standing, or ambulating: $41 \%$ vs $4 \%, P<.001) .53$

Another follow-up study from the Johns Hopkins medical ICU evaluated the safety of physical therapy interventions during 1,110 consecutive medical ICU admissions (60\% of which received mechanical ventilation) over a period of 53 months following completion of the quality improvement project. Of 5,267 physical therapy sessions, only $34(0.6 \%)$ had any potential safety event (all prospectively screened using standardized criteria). These events were mostly transient physiologic changes (eg, changes in mean arterial pressure and oxygen saturation) that resolved with rest. Less than 8 per 10,000 physical therapy sessions had an event that required any additional therapy, with no event requiring increased length of stay. ${ }^{34}$

A qualitative study conducted via independent semistructured interviews with 20 medical ICU staff reported that the Johns Hopkins quality improvement project resulted in an important shift in ICU culture, causing early mobility to be perceived as "common sense." In addition, 95\% of staff reported improved job satisfaction. Interviewees agreed that the components necessary for success of the quality improvement project included a supportive culture, the presence of a multidisciplinary team with good communication, a leader who could advocate for rehabilitation, and adequate resources (personnel, equipment, and funding). ${ }^{54}$ This quality improvement project serves as an important example of the steps needed to bridge the gap between research and practice, resulting in improved patient outcomes.

\section{Future Directions for the Field}

A number of technologies are being evaluated to assist with rehabilitation and mobilization of critically ill patients. A few such technologies will be mentioned here as part of future directions for the field.

Neuromuscular electrical stimulation is a rehabilitation modality that has been used extensively in physical medicine and rehabilitation practice. It delivers low-voltage electrical impulses through electrodes placed on the skin overlying target muscles, causing passive contraction. It is 
used in both in-patient and out-patient settings for the treatment of muscle weakness in patients with chronic disease, such as advanced COPD and congestive heart failure, ${ }^{55,56}$ and treatment of healthy athletes after sportsrelated injuries. ${ }^{57}$ In the critically ill population, there has been increasing interest in neuromuscular electrical stimulation for the prevention and treatment of ICU-acquired weakness, with several systematic reviews summarizing the evolving evidence. ${ }^{58-61}$ This promising therapy requires continued research before adoption into routine clinical care in the ICU setting.

Cycle ergometry uses a bedside device on which a supine patient can perform passive, active-assisted, or active in-bed cycling. Passive cycling may limit muscle protein catabolism in unconscious patients. ${ }^{62}$ In addition, this intervention does not appear to cause any clinically adverse hemodynamic or respiratory changes, even when applied early (within $72 \mathrm{~h}$ of starting mechanical ventilation). ${ }^{63}$ In a randomized controlled trial of 90 critically ill subjects with respiratory failure, cycle ergometry significantly increased quadriceps muscle strength, 6-min walking distance, and quality of life scores. ${ }^{64}$ A prospective study has demonstrated the safety and feasibility of cycle ergometry, as part of routine clinical practice, in the Johns Hopkins Hospital medical ICU. Over an 18-month period, 181 subjects $(80 \%$ mechanically ventilated) received a total of 541 cycling sessions with trained physical therapists, with only one safety event reported ( $0.2 \%$ rate) ${ }^{65}$

Functional electrical stimulation uses neuromuscular electrical stimulation to stimulate multiple groups of muscles concurrently in a functional way that mimics voluntary contraction. Functional electrical stimulation can be conducted with cycle ergometry to achieve functional electrical stimulation-assisted cycling. Results from a small pilot study indicate that functional electrical stimulationassisted cycling is safe and feasible, with potential benefit in enhancing functional recovery and reducing incidence of delirium. ${ }^{66}$ An ongoing multi-center randomized controlled trial (ClinicalTrials.gov identifier: NCT02214823) is being conducted at 4 sites in Australia and the United States to evaluate its effect on short- and long-term physical and cognitive outcomes in mechanically ventilated subjects. ${ }^{67}$ Other innovative therapies used in mechanically ventilated patients include hydrotherapy (mobilization in a swimming pool to overcome gravitational forces) ${ }^{68}$ and interactive video games. ${ }^{69}$

\section{Conclusions}

In modern day critical care, bed rest is common practice, especially for mechanically ventilated patients, with increasingly recognized short- and long-term negative sequelae. Early mobilization and rehabilitation is safe and feasible, with some evidence of improved patient outcomes, including decreased mechanical ventilation duration and improved physical functioning. Structured quality improvement projects are crucial for closing the large gap between these research findings and routine clinical practice, in order to expedite the post-ICU recovery of mechanically ventilated patients. Involving a multidisciplinary team, with a recognized leader, can be effective in changing ICU culture and practice to effectively deliver early mobilization and rehabilitation.

\section{REFERENCES}

1. Ries E. Some radical changes in the after-treatment of celiotomy cases. JAMA 1899;XXXIII(8):454-456. doi:10.1001/jama.1899. $92450600020001 \mathrm{~g}$.

2. Cunningham JH. Treatment of Prostatic Hypertrophy. Boston Med Surg J 1907;156(20):636-642.

3. Epstein HJ, Fleischer AJ. The disadvantages of the prolonged period of postpartum rest in bed. Am J Obstet Gynecol 1927;14(3):360-363.

4. Rock J. Progress in obstetrics. N Engl J Med 1929;200(18):919-927.

5. Bergel RR. Disabling effects of inactivity and importance of physical conditioning: a historical perspective. Rheum Dis Clin North Am 1990;16(4):791-801.

6. Keys A. Introduction to the symposium on convalescence and rehabilitation. Fed Proc 1944;3:189.

7. Powers JH. The abuse of rest as a therapeutic measure in surgery: early postoperative activity and rehabilitation. JAMA 1944;125(16): 1079-1083. doi: 10.1001/jama.1944.02850340005003.

8. Dock W. The evil sequelae of complete bed rest. JAMA 1944; 125(16):1083-1085. doi: 10.1001/jama.1944.02850340009004.

9. Ghormley RK. The abuse of rest in bed in orthopedic surgery. JAMA 1944;125(16):1085-1087. doi: 10.1001/jama.1944.02850340011005.

10. Foss G. A method for augmenting ventilation during ambulation. Phys Ther 1972;52(5):519-520.

11. Burns JR, Jones FL. Early ambulation of patients requiring ventilatory assistance. Chest 1975;68(4):608.

12. Petty TL. Suspended life or extending death? Chest 1998;114(2): 360-361.

13. Brower RG. Consequences of bed rest. Crit Care Med 2009;37(10 Suppl):S422-S428.

14. Suetta C, Hvid LG, Justesen L, Christensen U, Neergaard K, Simonsen L, et al. Effects of aging on human skeletal muscle after immobilization and retraining. J Appl Physiol 2009;107(4):1172-1180.

15. Jones SW, Hill RJ, Krasney PA, O'Conner B, Peirce N, Greenhaff PL. Disuse atrophy and exercise rehabilitation in humans profoundly affects the expression of genes associated with the regulation of skeletal muscle mass. FASEB J 2004;18(9):1025-1027.

16. English KL, Paddon-Jones D. Protecting muscle mass and function in older adults during bed rest. Curr Opin Clin Nutr Metab Care 2010;13(1):34-39.

17. Kortebein P, Ferrando A, Lombeida J, Wolfe R, Evans WJ. Effect of 10 days of bed rest on skeletal muscle in healthy older adults. JAMA 2007;297(16):1772-1774.

18. Puthucheary ZA, Rawal J, McPhail M, Connolly B, Ratnayake G, Chan $\mathrm{P}$, et al. Acute skeletal muscle wasting in critical illness. JAMA 2013;310(15):1591-1600.

19. Derde S, Hermans G, Derese I, Güiza F, Hedström Y, Wouters PJ, et al. Muscle atrophy and preferential loss of myosin in prolonged critically ill patients. Crit Care Med 2012;40(1):79-89.

20. Fan E, Dowdy DW, Colantuoni E, Mendez-Tellez PA, Sevransky JE, Shanholtz C, et al. Physical complications in acute lung injury sur- 


\section{Early Mobilization and Rehabilitation IN the ICU}

vivors: a two-year longitudinal prospective study. Crit Care Med 2014;42(4):849-859.

21. Stevens RD, Marshall SA, Cornblath DR, Hoke A, Needham DM, De Jonghe B, et al. A framework for diagnosing and classifying intensive care unit-acquired weakness. Crit Care Med 2009;37(10 Suppl):S299-S308.

22. De Jonghe B, Sharshar T, Lefaucheur JP, Authier FJ, DurandZaleski I, Boussarsar M, et al. Paresis acquired in the intensive care unit: a prospective multicenter study. JAMA 2002;288(22):2859-2867.

23. Kleyweg RP, van der Meché FG, Schmitz PI. Interobserver agreement in the assessment of muscle strength and functional abilities in Guillain-Barre syndrome. Muscle Nerve 1991;14(11):1103-1109.

24. Hermans G, De Jonghe B, Bruyninckx F, Van den Berghe G. Clinical review: critical illness polyneuropathy and myopathy. Crit Care 2008;12(6):238.

25. Denehy L, Skinner EH, Edbrooke L, Haines K, Warrillow S, Hawthorne $\mathrm{G}$, et al. Exercise rehabilitation for patients with critical illness: a randomized controlled trial with 12 months of followup. Crit Care 2013;17(4):R156.

26. De Jonghe B, Bastuji-Garin S, Sharshar T, Outin H, Brochard L. Does ICU-acquired paresis lengthen weaning from mechanical ventilation? Intensive Care Med 2004;30(6):1117-1121.

27. De Jonghe B, Bastuji-Garin S, Durand MC, Malissin I, Rodrigues P, Cerf C, et al. Respiratory weakness is associated with limb weakness and delayed weaning in critical illness. Crit Care Med 2007;35(9): 2007-2015.

28. Hermans G, Van Mechelen H, Clerckx B, Vanhullebusch T, Mesotten $\mathrm{D}$, Wilmer A, et al. Acute outcomes and 1-year mortality of intensive care unit-acquired weakness: a cohort study and propensity-matched analysis. Am J Respir Crit Care Med 2014;190(4):410-420.

29. Fan E, Cheek F, Chlan L, Gosselink R, Hart N, Herridge MS, et al. An official American Thoracic Society Clinical Practice guideline: the diagnosis of intensive care unit-acquired weakness in adults. Am J Respir Crit Care Med 2014;190(12):1437-1446.

30. Wieske L, Dettling-Ihnenfeldt DS, Verhamme C, Nollet F, van Schaik IN, Schultz MJ, et al. Impact of ICU-acquired weakness on post-ICU physical functioning: a follow-up study. Crit Care 2015;19:196.

31. Herridge MS, Cheung AM, Tansey CM, Matte-Martyn A, DiazGranados N, Al-Saidi F, et al. One-year outcomes in survivors of the acute respiratory distress syndrome. N Engl J Med 2003;348(8):683693.

32. Herridge MS, Tansey CM, Matté A, Tomlinson G, Diaz-Granados $\mathrm{N}$, Cooper A, et al. Functional disability 5 years after acute respiratory distress syndrome. N Engl J Med 2011;364(14):1293-1304.

33. Nydahl P, Ruhl AP, Bartoszek G, Dubb R, Filipovic S, Flohr HJ, et al. Early mobilization of mechanically ventilated patients: a 1-day point-prevalence study in Germany. Crit Care Med 2014;42(5):11781186.

34. Sricharoenchai T, Parker AM, Zanni JM, Nelliot A, Dinglas VD, Needham DM. Safety of physical therapy interventions in critically ill patients: a single-center prospective evaluation of 1110 intensive care unit admissions. J Crit Care 2014;29(3):395-400.

35. Berney SC, Harrold M, Webb SA, Seppelt I, Patman S, Thomas PJ, Denehy L. Intensive care unit mobility practices in Australia and New Zealand: a point prevalence study. Crit Care Resusc 2013; 15(4):260-265.

36. Bailey P, Thomsen GE, Spuhler VJ, Blair R, Jewkes J, Bezdjian L, et al. Early activity is feasible and safe in respiratory failure patients. Crit Care Med 2007:35(1):139-145.

37. Hodgson CL, Stiller K, Needham DM, Tipping CJ, Harrold M, Baldwin $\mathrm{CE}$, et al. Expert consensus and recommendations on safety criteria for active mobilization of mechanically ventilated critically ill adults. Crit Care 2014;18(6):658.
38. Morandi A, Brummel NE, Ely EW. Sedation, delirium and mechanical ventilation: the "ABCDE" approach. Curr Opin Crit Care 2011; 17(1):43-49.

39. Balas MC, Vasilevskis EE, Olsen KM, Schmid KK, Shostrom V, Cohen MZ, et al. Effectiveness and safety of the awakening and breathing coordination, delirium monitoring/management, and early exercise/mobility bundle. Crit Care Med 2014;42(5):1024-1036.

40. Morris PE, Goad A, Thompson C, Taylor K, Harry B, Passmore L, et al. Early intensive care unit mobility therapy in the treatment of acute respiratory failure. Crit Care Med 2008;36(8):2238-2243.

41. Morris PE, Griffin L, Berry M, Thompson C, Hite RD, Winkelman $\mathrm{C}$, et al. Receiving early mobility during an intensive care unit admission is a predictor of improved outcomes in acute respiratory failure. Am J Med Sci 2011;341(5):373-377.

42. Schweickert WD, Pohlman MC, Pohlman AS, Nigos C, Pawlik AJ, Esbrook CL, et al. Early physical and occupational therapy in mechanically ventilated, critically ill patients: a randomised controlled trial. Lancet 2009;373(9678):1874-1882.

43. Moss M, Nordon-Craft A, Malone D, Van Pelt D, Frankel SK, Warner ML, et al. A randomized trial of an intensive physical therapy program for acute respiratory failure patients. Am J Respir Crit Care Med 2015 [Epub ahead of print].

44. Jolley SE, Moss M, Needham DM, Caldwell ES, Morris, PE, Miller, $\mathrm{RR}$, et al. Point prevalence study of intensive care unit mobility across the acute respiratory distress syndrome network: moving the needle on ICU-associated neuromuscular weakness (abstract). American Thoracic Society International Conference Abstracts 2015: A6349.

45. Malone D, Ridgeway K, Nordon-Craft A, Moss P, Schenkman M, Moss M. Physical therapist practice in the intensive care unit: results of a national survey. Phys Ther 2015;95(10):1335-1344.

46. Bakhru RN, Wiebe DJ, McWilliams DJ, Spuhler VJ, Schweickert WD. An environmental scan for early mobilization practices in U.S. ICUs. Crit Care Med 2015;43(11):2360-2369.

47. TEAM Study Investigators, Hodgson C, Bellomo R, Berney S, Bailey $\mathrm{M}$, Buhr H, et al. Early mobilization and recovery in mechanically ventilated patients in the ICU: a bi-national, multi-centre, prospective cohort study. Crit Care 2015;19:81.

48. Leditschke IA, Green M, Irvine J, Bissett B, Mitchell IA. What are the barriers to mobilizing intensive care patients? Cardiopulm Phys Ther J 2012;23(1):26-29.

49. Pronovost PJ, Berenholtz SM, Needham DM. Translating evidence into practice: a model for large scale knowledge translation. BMJ 2008;337:a1714.

50. Needham DM, Korupolu R. Rehabilitation quality improvement in an intensive care unit setting: implementation of a quality improvement model. Top Stroke Rehabil 2010;17(4):271-281.

51. Needham DM, Korupolu R, Zanni JM, Pradhan P, Colantuoni E, Palmer JB, et al. Early physical medicine and rehabilitation for patients with acute respiratory failure: a quality improvement project. Arch Phys Med Rehabil 2010;91(4):536-542.

52. Hager DN, Dinglas VD, Subhas S, Rowden AM, Neufeld KJ, Bienvenu OJ, et al. Reducing deep sedation and delirium in acute lung injury patients: a quality improvement project. Crit Care Med 2013; 41(6):1435-1442.

53. Dinglas VD, Parker AM, Reddy DR, Colantuoni E, Zanni JM, Turnbull AE, et al. A quality improvement project sustainably decreased time to onset of active physical therapy intervention in patients with acute lung injury. Ann Am Thorac Soc 2014;11(8):1230-1238.

54. Eakin MN, Ugbah L, Arnautovic T, Parker AM, Needham DM. Implementing and sustaining an early rehabilitation program in a medical intensive care unit: a qualitative analysis. J Crit Care 2015; 30(4):698-704. 


\section{Early Mobilization and Rehabilitation IN the ICU}

55. de Oliveira Melo M, Aragão FA, Vaz MA. Neuromuscular electrical stimulation for muscle strengthening in elderly with knee osteoarthritis: a systematic review. Complement Ther Clin Pract 2013;19(1): 27-31.

56. Maddocks M, Gao W, Higginson IJ, Wilcock A. Neuromuscular electrical stimulation for muscle weakness in adults with advanced disease. Cochrane Database Syst Rev 2013;(1):CD009419.

57. Lam KC, Snyder Valier AR, Valovich McLeod TC. Injury and treatment characteristics of sport-specific injuries sustained in interscholastic athletics: a report from the athletic training practice-based research network. Sports Health 2015;7(1):67-74.

58. Segers J, Hermans G, Bruyninckx F, Meyfroidt G, Langer D, Gosselink R. Feasibility of neuromuscular electrical stimulation in critically ill patients. J Crit Care 2014;29(6):1082-1088.

59. Wageck B, Nunes GS, Silva FL, Damasceno MC, de Noronha M. Application and effects of neuromuscular electrical stimulation in critically ill patients: systematic review. Med Intensiva 2014;38(7): 444-454.

60. Williams N, Flynn M. A review of the efficacy of neuromuscular electrical stimulation in critically ill patients. Physiother Theory Pract 2014;30(1):6-11.

61. Kho ME, Truong AD, Zanni JM, Ciesla ND, Brower RG, Palmer JB, Needham DM. Neuromuscular electrical stimulation in mechanically ventilated patients: a randomized, sham-controlled pilot trial with blinded outcome assessment. J Crit Care 2015;30(1):32-39.

62. Preiser J, Prato C, Harvengt A, Peters L, Bastin M. Passive cycling limits myofibrillar protein catabolism in unconscious patients: a pilot study. J Nov Physiother 2014;4:225. doi: 10.4172/2165-7025.1000225.
63. Camargo Pires-Neto R, Fogaça Kawaguchi YM, Sayuri Hirota A, Fu $\mathrm{C}$, Tanaka $\mathrm{C}$, Caruso $\mathrm{P}$, et al. Very early passive cycling exercise in mechanically ventilated critically ill patients: physiological and safety aspects: a case series. PLoS One 2013;8(9):e74182.

64. Burtin C, Clerckx B, Robbeets C, Ferdinande P, Langer D, Troosters $\mathrm{T}$, et al. Early exercise in critically ill patients enhances short-term functional recovery. Crit Care Med 2009;37(9):2499-2505.

65. Kho ME, Martin RA, Toonstra AL, Zanni JM, Mantheiy EC, Nelliot A, Needham DM. Feasibility and safety of in-bed cycling for physical rehabilitation in the intensive care unit. J Crit Care 2015;30(6): 1419.e1-e5.

66. Parry SM, Berney S, Warrillow S, El-Ansary D, Bryant AL, Hart N, et al. Functional electrical stimulation with cycling in the critically ill: a pilot case-matched control study. J Crit Care 2014;29(4):695697.e1-e7.

67. Parry SM, Berney S, Koopman R, Bryant A, El-Ansary D, Puthucheary Z, et al. Early rehabilitation in critical care (eRiCC): functional electrical stimulation with cycling protocol for a randomised controlled trial. BMJ Open 2012;2(5):e001891. doi: 10.1136/bmjopen2012-001891

68. Felten-Barentsz KM, Haans AJ, Slutsky AS, Heunks LM, van der Hoeven JG. Feasibility and safety of hydrotherapy in critically ill ventilated patients. Am J Respir Crit Care Med 2015;191(4): 476-477.

69. Kho ME, Damluji A, Zanni JM, Needham DM. Feasibility and observed safety of interactive video games for physical rehabilitation in the intensive care unit: a case series. J Crit Care 2012;27(2):219.e1-e6. 